\title{
INFLUENCE DES TRAITEMENTS MÉCANIQUES ET HYDROTHERMIQUES SUR LA VALEUR NUTRITIVE DU MAÏS POUR LES JEUNES BOVINS A L'ENGRAIS
}

\author{
C. BÉRANGER, P. THIVEND et R. JARRIGE \\ avec la collaboration technique de G. Cuysle \\ Station de Recherches sur l'Élevage des Ruminants, \\ Centre de Recherches de Clermont-Ferrand, I. N. R. A., \\ 63 - Saint-Genès-Champanelle
}

\section{RÉSUMÉ}

Quatre expériences d'engraissement de jeunes bovins ont été effectuées pour étudier l'influence de trois traitements technologiques sur la valeur nutritive du mais : broyage fin suivi d'un pressage (expérience 1 ), cuisson à l'eau bouillante pendant 30 ou $45 \mathrm{mn}$ (expériences 2 et 3) et trempage pendant 48 heures suivi d'un séchage sur rouleaux à $70^{\circ} \mathrm{C}$ (expérience 4). Dans chaque cas, le lot expérimental recevant le maiss traité a été comparé à un lot témoin recevant un maïs grossièrement moulu.

I 26 jeunes bovins ont été engraissés pendant des périodes de Ioo à I 50 jours. Le maïs a été distribué à tous les animaux, à raison de I à I, $5 \mathrm{~kg}$ de matière sèche par roo $\mathrm{kg}$ de poids vif; la quantité de foin distribuée était voisine du niveau maximum d'ingestion, mais limitée à 50 p. Ioo de la matière sèche de la ration.

Au cours des expériences $I, 2$ et 4 , on n'a pas noté d'influence significative du traitement sur le gain de poids vif et de carcasse, sur l'état d'engraissement et sur l'efficacité alimentaire. Au cours de la première période de l'expérience 3 (foin de luzerne sous forme normale), les animaux recevant le maïs traité ont eu une vitesse de croissance plus élevée et une meilleure efficacité alimentaire $(\mathrm{P}<\mathrm{o}, \mathrm{oI})$ que les animaux du lot témoin. Les différences se sont atténuées au cours de la deuxième période (luzerne déshydratée et condensée).

La cuisson n'a pas eu d'influence sur la digestibilité des principaux constituants de la ration. Bien que les trois traitements étudiés aient modifié la structure de l'amidon, les fermentations du rumen n'ont pas été orientées dans un sens favorable à une meilleure utilisation de l'énergie. $\mathrm{Si}$ ces résultats ne mettent pas clairement en évidence des différences significatives, ils traduisent cependant, dans chaque expérience, une légère amélioration de la valeur nutritive du mais lorsqu'il est traité.

\section{INTRODUCTION}

Les amidons de la ration du ruminant sont en majeure partie transformés par les microorganismes du rumen en acides gras volatils et en substance microbienne; le reste, digéré dans l'inte stin grêle, est absorbé sous forme de glucose. 


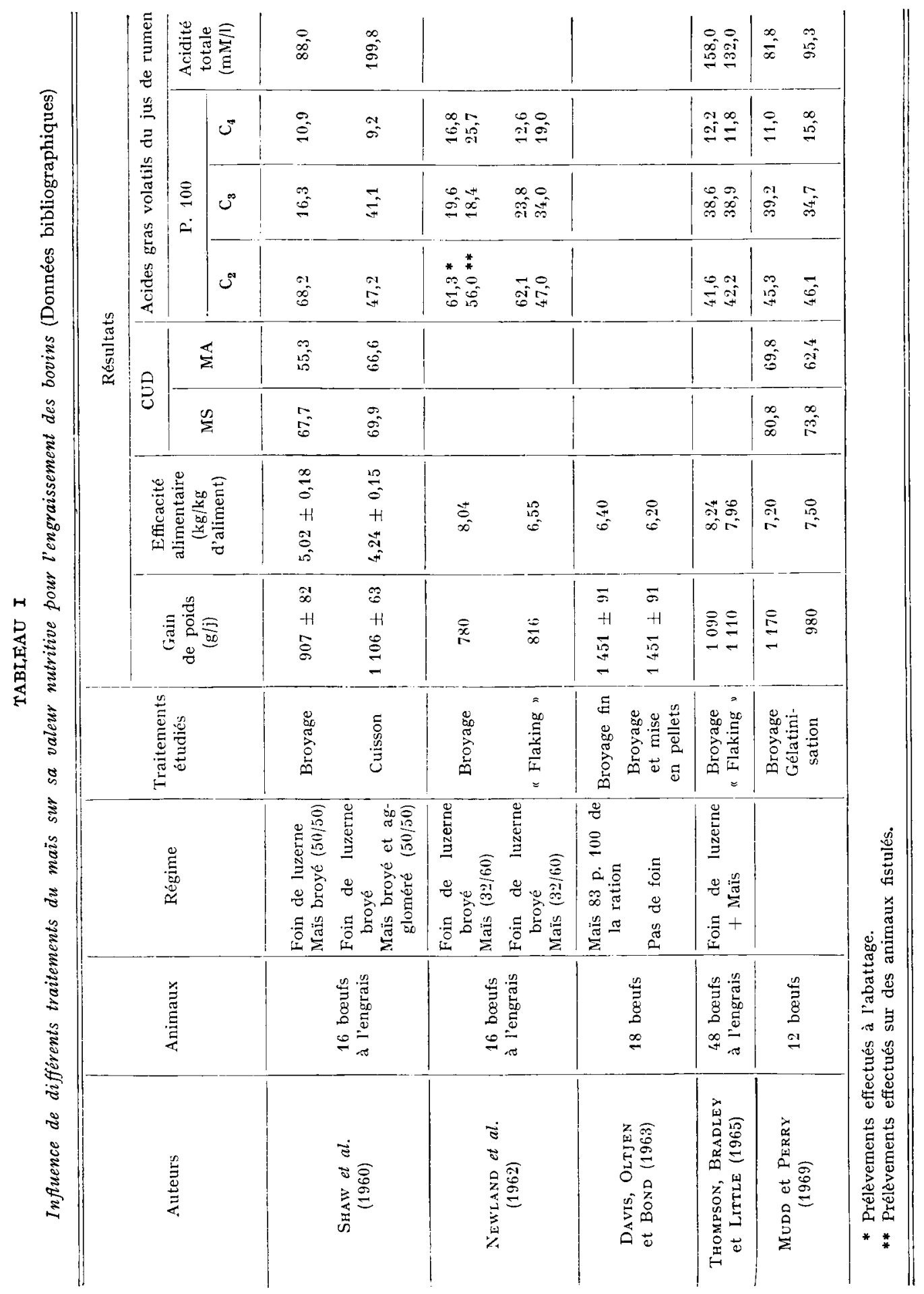


Il semble possible de modifier les proportions respectives de ces différents produits terminaux en faisant subir à la céréale certains traitements industriels. Ceux-ci provoqueraient une amélioration de l'utilisation de l'énergie en favorisant la formation d'acide propionique (PHIL LIPSON, r952) qui est mieux utilisé pour l'engraissement que l'acide acétique (Armstrong et Blaxter, I96I). A parcir de ces données, de nombreux travaux américains ont été entrepris pour essayer d'accroitre 1'utilisation digestive et métabolique du maïs par le ruminant (ENSOR, SHAw et TELLECHEA, I959; SHAW et al., I960; NEWLAND et al., I962; WoOds et LUTHFR, I962 ; Johnson, I967; MUDD et PERRY, I969). Les différents traitements technologiques étudiés ont provoqué généralement une amélioration de l'efficacité alimentaire de la ration (tabl. I), soit en augmentant la vitesse de croissance des animaux, soit le plus souvent en diminuant les quantités consommées. Cette amélioration peut s'expliquer par une digestibilité plus importante (Johnson, Marshushima et KNox, I968), par une rétention azotée accrue (ARNE'TT et BRADLEY, I96r) ou par une meilleure utilisation énergétique des produits terminaux de la digestion de l'amidon traité.

Cependant, les traitements mécaniques ou hydrothermiques étudiés sont généralement trop complexes pour être préconisés dans la pratique. C'est la raison pour laquelle nous avons étudié l'influence de trois traitements simples sur la valeur nutritive du maïs distribué à de jeunes bovins à l'engrais. Il s'agit du broyage fin (grille de $3 \mathrm{~mm}$ ) suivi d'un pressage, de la cuisson à l'eau bouillante pendant 30 ou $45 \mathrm{mn}$, ou du trempage pendant 48 heures suivi d'un séchage sur rouleaux à $70^{\circ} \mathrm{C}$.

De I963 à I966, nous avons réalisé quatre expériences sur des bouvillons ou des tautillons abattus à un âge allant de I4 à I 8 mois. Dans chaque cas, le lot expérimental recevant le maîs traité était comparé à un lot témoin recevant un maîs grossièrement moulu; le maìs représentait environ $50 \mathrm{p}$. Ioo de la ration, qui comportait en outre du foin et une certaine quantité de tourteau. La nature et la qualité du foin ainsi que l'ordre de distribution des différents aliments ont varié selon les expériences. Dans chaque expérience, on a mesuré l'ingestion journalière, la vitesse de croissance des animaux, le poids et l'état d'engraissement de la carcasse. Dans certains cas, on a mesuré la digestibilité de la ration et la composition du mélange d'acides gras volatils du jus de rumen.

\section{MATÉRIEL, E'T MÉTHODES}

Animaux

Nous avons utilisé I 26 animaux, âgés de 9 à I 2 mois au début des expériences, qui ont été engraissés pendant des périodes de Ioo à I 50 jours. Il s'agissait de bouvillons dans les expériences $\mathrm{I}$ et 2 et de taurillons dans les expériences 2, 3 et 4 . Ces animaux étaient de race précoce (Frisonne, Normande) ou tardive (Charolaise, Salers $\times$ Charolais). Au début de chaque expérience, les animaux ont été appariés en fonction de leur poids vif, de leur âge, de leur race et de leur état d'engraissement.

\section{Alimentation}

Les différents régimes distribués au cours des quatre expériences ainsi que leur mode de distribution sont décrits au tableau 2. Dans chaque expérience, 12 animaux de chaque lot ont été alimentés individuellement et les autres, en lot, par groupe de 5 ou 6 . Le maìs était distribué 


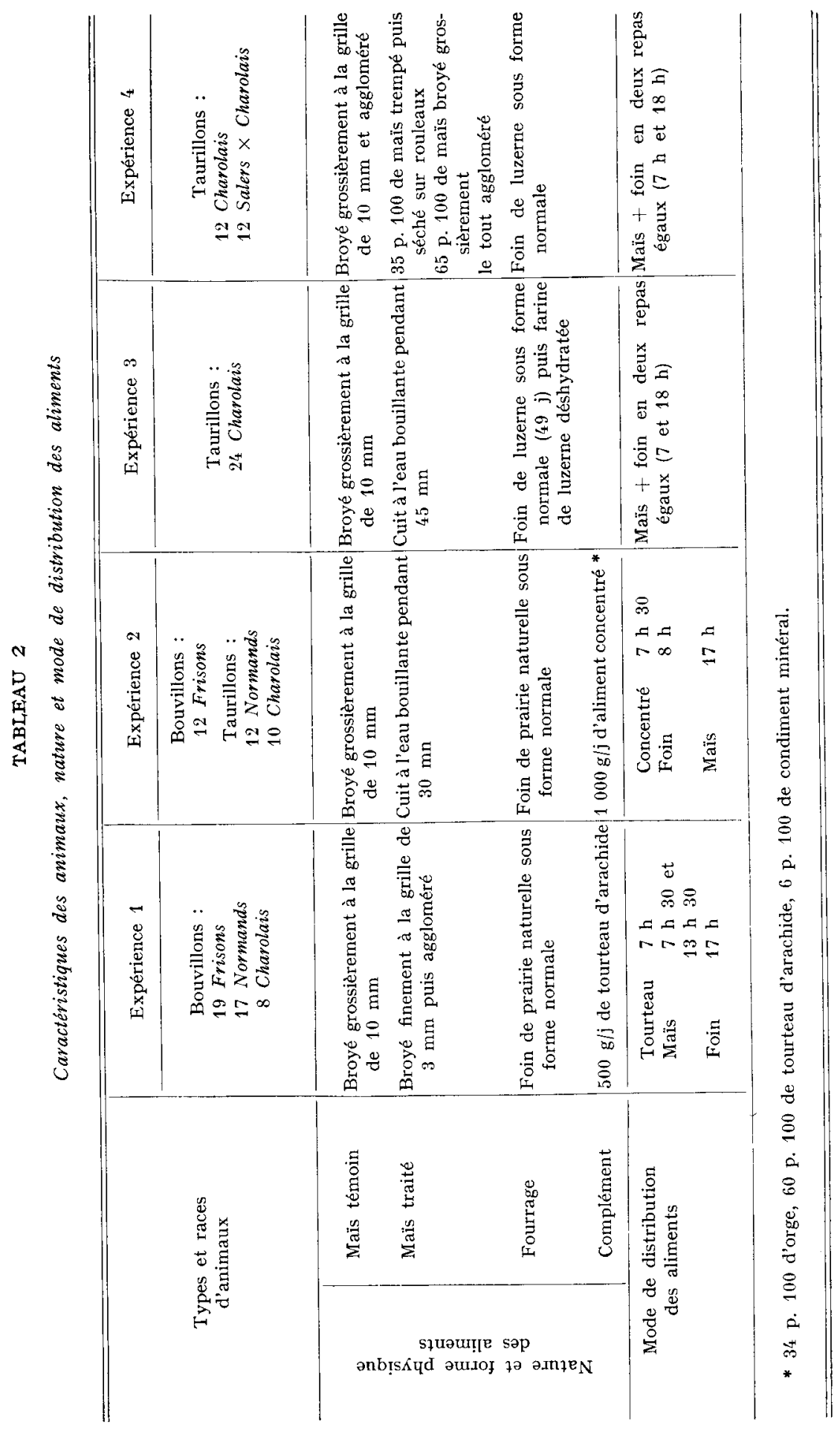


en quantité limitée, identique dans les deux lots à raison de I à $\mathbf{I}, 5 \mathrm{~kg}$ de matière sèche par I $00 \mathrm{~kg}$ de poids vif. La quantité de foin distribuée était voisine du niveau maximum d'ingestion déterminé pendant une prépériode de I $5_{5}$ jours; elle augmentait ensuite avec le poids des animaux sans dépasser toutefois $50 \mathrm{p}$. Ioo de la matière sèche de la ration. Les animaux recevaient en outre $500 \mathrm{~g}$ de tourteau d'arachide dans l'expérience $\mathrm{I}$ et dans la $\mathrm{I}^{\mathrm{re}}$ période de l'expérience 3 et I $\mathrm{kg}$ d'aliment concentré dans l'expérience 2 . Ils pouvaient consommer la paille de leur litière. On a mesuré les quantités d'aliments distribuées et éventuellement refusées pendant 6 jours par semaine.

\section{TABLEAU 3}

Composition des aliments distribués

(p. Ioo de la matière sèche)

\begin{tabular}{|c|c|c|c|c|c|}
\hline Expérience & \multicolumn{2}{|c|}{ Nature de l'aliment } & $\begin{array}{l}\text { Cellulose } \\
\text { brute } \\
\text { Weende }\end{array}$ & $\begin{array}{l}\text { Matières } \\
\text { azotées }\end{array}$ & Cendres \\
\hline \multirow{5}{*}{1} & \multirow{2}{*}{ Maïs } & 1 & 2,9 & 9,8 & 2,6 \\
\hline & & 2 & 2,8 & 10,3 & 2,8 \\
\hline & \multirow{2}{*}{$\begin{array}{l}\text { Foin de prairie } \\
\text { naturelle }\end{array}$} & $a$ & 28,2 & 11,9 & 6,8 \\
\hline & & $b$ & 30,9 & 9,7 & 7,6 \\
\hline & \multicolumn{2}{|c|}{ Tourteau } & 6,4 & 62,8 & 5,1 \\
\hline \multirow{4}{*}{2} & \multirow{2}{*}{ Maïs } & 1 & 3,8 & 10,9 & 3,5 \\
\hline & & 2 & 4,7 & 10,5 & 3,4 \\
\hline & \multicolumn{2}{|c|}{ Foin de prairie naturelle } & 35,1 & 8,1 & 8,1 \\
\hline & \multicolumn{2}{|c|}{ Aliment concentré } & 6,5 & 39,6 & 7,9 \\
\hline \multirow{5}{*}{3} & \multirow{2}{*}{ Maïs } & 1 & 2,2 & 11,0 & 1,8 \\
\hline & & 2 & & 11,6 & 1,5 \\
\hline & \multicolumn{2}{|c|}{ Foin de luzerne normal } & 29,3 & 17,4 & 8,4 \\
\hline & \multicolumn{2}{|c|}{ Foin de luzerne aggloméré } & 31,8 & 16,5 & 9,2 \\
\hline & \multicolumn{2}{|c|}{ Tourteau } & 4,9 & 56,3 & 6,5 \\
\hline \multirow{3}{*}{4} & \multirow{2}{*}{ Maïs } & 1 & 3,7 & 10,4 & 2,9 \\
\hline & & 2 & 4,0 & 9,4 & 3,0 \\
\hline & \multicolumn{2}{|c|}{ Foin de luzerne } & 37,4 & 16,6 & 7,8 \\
\hline
\end{tabular}

1 : Maïs témoin. - 2 : Maïs traité.

$a$ : Distribué du 10-12-62 au 18-3-63.

$b$ : Distribué du 19-3-63 au 19-5-63.

Le tableau 3 donne la composition des aliments distribués. Les foins de prairie naturelle utilisés au cours des deux premiers essais étaient pauvres en azote; en revanche, dans les deux 
autres essais, le foin de luzerne était de bonne qualité. Les traitements hydrothermiques n'ont pas modifié la composition chimique de la céréale sauf dans le cas du dernier essai pour lequel la teneur en matières azotées du maïs traité a été légèrement plus faible que celle du maïs témoin.

\section{Croissance et abattage}

Les animaux ont été pesés pendant 3 jours consécutifs à I 4 heures, au début et à la fin de la période expérimentale ainsi que pendant les 3 jours précédant l'abattage. Ce sont les moyennes de ces pesées qui ont été utilisées pour calculer les gains de poids vif moyens. Les animaux ont été également pesés une fois par quinzaine pour étudier l'évolution de la vitesse de croissance pendant l'expérience.

Dans chaque essai, les animaux ont été abattus par couple, dès que l'un d'eux avait atteint un poids et un état d'engraissement suffisants. On a mesuré le poids de la carcasse chaude, le rendement, le poids des contenus digestifs, l'état d'engraissement à partir du poids des dépôts adipeux périrénaux et précruraux et du pourcentage de dépôts adipeux de la I I e côte (GEAY et BÉRANGER, I969).

\section{Digestibilité}

Au cours du premier essai, nous avons mesuré la digestibilité globale des deux rations sur cinq animaux de chaque lot, en utilisant l'oxyde de chrome comme marqueur (Christian et Covp, I954). Pendant 20 jours, on a distribué quotidiennement $20 \mathrm{~g}$ de $\mathrm{Cr}_{2} \mathrm{O}_{3}$ dans un $\mathrm{kg}$ de maîs. Durant les ro derniers jours, on a effectué 4 prélèvements de fèces par jour (8, II, I 4 et 17 heures) sur lesquels on a dosé l'oxyde de chrome.

Au cours du deuxième essai, nous avons étudié sur 4 animaux (deux couples de jumeaux) maintenus en cage à bilan, la digestibilité des principaux constituants des deux rations et la rétention azotée. Les mesures ont été effectuées pendant deux périodes de r 2 jours chacune.

\section{Étude des produits terminaux de la digestion}

Nous avons mesuré l'acidité totale du jus de rumen et sa composition en acides gras volatils par la méthode de JAmes et MARTin (I95I) sur des échantillons prélevés, soit par tubage sur quelques animaux de chaque lot au cours de la période expérimentale, soit sur deux animaux porteurs d'une canule du rumen, recevant les régimes étudiés. Les prélèvements par tubage ont été effectués 3 ou I 3 heures après le début du repas; ceux effectués sur les animaux fistulés ont été plus fréquents et nous ont permis d'établir l'évolution de la concentration des produits terminaux de la digestion dans le rumen.

\section{RÉSULTATS}

\section{Influence de la finesse de broyage et du pressage (expérience $\mathrm{n}^{\circ} \mathrm{I}$ )}

Dans cet essai réalisé sur 44 bouvillons, le lot expérimental a reçu du maïs finement broyé (grille à mailles de $3 \mathrm{~mm}$ de diamètre) et aggloméré dans une presse à filière de Io $\mathrm{mm}$ de diamètre (température de $1^{\prime}$ ordre de $60^{\circ} \mathrm{C}$ ). Le gain de poids est resté inférieur à I ooo $\mathrm{g} / \mathrm{j}$ parce que les animaux étaient castrés et avaient une ingestion relativement faible pour éviter les troubles digestifs ( $\mathrm{I}, 83$ et $\mathrm{I}, 89 \mathrm{~kg}$ de matière sèche par Ioo $\mathrm{kg}$ de poids vif). Ils ont produit des carcasses légères et relativement grasses.

On n'a pas noté de différence significative entre les deux régimes, que ce soit pour le gain de poids vif et de carcasse, l'état d'engraissement ou l'efficacité alimentaire (tabl. 4). Il en a été de même pour les proportions respectives des acides gras volatils dans le rumen (tabl. 8) bien que l'acidité totale du jus ait été plus impor- 
tante dans le cas du maïs granulé. Cependant, la ration expérimentale contenant le maïs finement broyé et aggloméré a été plus digestible : $72 \mathrm{p}$. Ioo pour la matière sèche au lieu de $68 \mathrm{p}$. Ioo pour la ration témoin.

\section{TABLEAU 4}

Expérience no 1: comparaison de la valeur nutritive du maïs grossièrement broyé et du mais broyé finement et aggloméré

\begin{tabular}{|c|c|c|}
\hline & $\begin{array}{l}\text { Maïs } \\
\text { grossièrement } \\
\text { broyé }\end{array}$ & $\begin{array}{l}\text { Maïs broyé } \\
\text { finement et } \\
\text { aggloméré }\end{array}$ \\
\hline Nombre d'animaux $\ldots \ldots \ldots \ldots \ldots \ldots$ & 22 & 22 \\
\hline Durée de la période expérimentale (j) ... & 143 & 143 \\
\hline Poids initial $(\mathrm{kg}) \ldots \ldots \ldots \ldots \ldots \ldots$ & 309 & 310 \\
\hline Poids final $(\mathrm{kg}) \ldots \ldots \ldots \ldots \ldots \ldots$ & 428 & 435 \\
\hline Gain de poids journalier $(\mathrm{g}) \quad \ldots \ldots \ldots \ldots$ & $875 \pm 104$ & $918 \pm 151$ \\
\hline 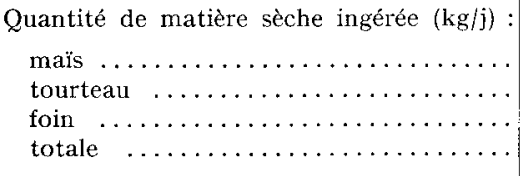 & $\begin{array}{l}3,18 \\
0,44 \\
3,4 \mathbf{4} \\
7,06\end{array}$ & $\begin{array}{l}3,31 \\
0,44 \\
3,21 \\
6,96\end{array}$ \\
\hline $\begin{array}{l}\text { Quantité de matière sèche consommée : } \\
\text { par } 100 \mathrm{~kg} \text { de poids vif } \ldots \ldots \ldots \ldots \ldots \\
\text { par } \mathrm{kg} \text { de gain } \ldots \ldots \ldots \ldots \ldots \ldots \ldots\end{array}$ & $\begin{array}{l}1,89 \\
8,12\end{array}$ & $\begin{array}{l}1,83 \\
7,75\end{array}$ \\
\hline Poids de carcasse chaude $(\mathrm{kg}) \ldots \ldots \ldots \ldots$ & $247 \pm 17$ & $248 \pm 20$ \\
\hline$\frac{\text { Poids de carcasse chaude }}{\text { Poids vif vide }} \times 100 \ldots \ldots$ & $68,1 \pm 0,7$ & $67,5 \pm 1,2$ \\
\hline $\begin{array}{r}\text { Dépôts adipeux périrénaux (p. } 100 \text { du } \\
\text { poids de carcasse) } \ldots \ldots \ldots \ldots \ldots \ldots \ldots\end{array}$ & $3,37 \pm 1,0$ & $3,20 \pm 0,9$ \\
\hline
\end{tabular}

\section{Infuence de la cuisson (expériences $\mathrm{n}^{\circ} 2$ et $\mathrm{n}^{\circ} 3$ )}

Nous avons pensé que les faibles différences observées dans l'expérience précédente étaient dues au fait que le traitement appliqué au maïs n'avait guère modifié la structure du grain d'amidon. Pour cette raison, nous avons étudié dans les deux essais suivants, l'influence d'un traitement hydrothermique plus sévère que le précédent, modifiant l'amidon de façon importante. Nous avons comparé au maìs normal grossièrement moulu, le maïs cuit à l'eau bouillante et distribué humide.

\section{Expérience $n^{\circ} 2$ (tab1. 5).}

Chaque lot de $\mathrm{I} 7$ animaux comprenait cette fois des animaux de type différent: 6 bouvillons Frisons en stabulation libre, 6 taurillons Normands et 5 taurillons Charolais en stabulation entravée. Ils recevaient, outre le maïs ( $\mathrm{I}$ à I,2 p. Ioo du poids vif), du foin de prairie naturelle, de qualité médiocre ( $\mathrm{p}$. Ioo du poids vif) qu'ils ont consommé en faible quantité $(2,5$ à $3,5 \mathrm{~kg}$ de matière sèche par jour) et un $\mathrm{kg}$ d'aliment concentré à $40 \mathrm{p}$. Ioo de matières azotées. Les animaux du lot expérimental recevaient une quantité de matière sèche de maïs cuit correspondant à celle du maîs cru distribué aux animaux du lot témoin. Le maîs cuit était obtenu 
en mélangeant le maïs entier à 2 fois son poids d'eau froide, puis en portant le mélange à ébullition et en maintenant la température penđant 30 minutes. L'eau en excès était soutirée ; elle représentait $34 \mathrm{p}$. Ioo de l'eau introduite et contenait 4,5 p. Ioo de matière sèche. On laissait le maïs cuit refroidir pendant I2 heures.

\section{TABLEAU 5}

Expérience no 2: comparaison de la valeur nutritive du mais grossièrement broyé et du mais cuit

\begin{tabular}{|c|c|c|c|c|c|c|}
\hline \multirow{2}{*}{ Races } & \multicolumn{2}{|c|}{ Normands } & \multicolumn{2}{|c|}{ Charolais } & \multicolumn{2}{|c|}{ Frisons } \\
\hline & $\begin{array}{c}1 \\
\text { témoin }\end{array}$ & $\stackrel{2}{2}$ & $\begin{array}{c}1 \\
\text { témoin }\end{array}$ & $\stackrel{2}{\text { traité }}$ & $\begin{array}{c}1 \\
\text { témoin }\end{array}$ & $\stackrel{2}{\text { traité }}$ \\
\hline Nombre d'animaux $\ldots \ldots \ldots \ldots \ldots \ldots$ & 6 & 6 & 5 & 5 & 6 & 6 \\
\hline Durée de la période expérimentale $(\mathrm{j}) \ldots$ & 159 & 159 & 147 & 147 & 147 & 147 \\
\hline Poids initial $(\mathrm{kg}) \ldots \ldots \ldots \ldots \ldots \ldots \ldots$ & 243 & 245 & 326 & 348 & 318 & 320 \\
\hline Poids final $(\mathrm{kg}) \ldots \ldots \ldots \ldots \ldots \ldots \ldots$ & 409 & 415 & 493 & 514 & 436 & 454 \\
\hline Gain de poids journalier $(g) \ldots \ldots \ldots \ldots$ & $\begin{array}{l}1039 \\
\pm 100\end{array}$ & $\begin{array}{l}1072 \\
\pm 73\end{array}$ & $\begin{array}{l}1133 \\
\pm 108\end{array}$ & $\begin{array}{l}1125 \\
\pm 155\end{array}$ & $\begin{array}{l}798 \\
\pm 62\end{array}$ & $\begin{array}{l}909^{*} \\
\pm 71\end{array}$ \\
\hline Quantité de matière sèche ingérée $(\mathrm{kg} / \mathrm{j})$ : & & & & & & \\
\hline 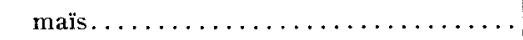 & 2,93 & 2,90 & 3,88 & 3,82 & 3,51 & 3,55 \\
\hline aliment concentré $\ldots \ldots \ldots \ldots \ldots$ & 0,87 & 0,87 & 0,61 & 0,80 & 0,89 & 0,89 \\
\hline foin $\ldots \ldots \ldots \ldots \ldots \ldots \ldots \ldots \ldots$ & 2,60 & 2,37 & 2,86 & 2,98 & 3,22 & 3,20 \\
\hline totale $\ldots \ldots \ldots \ldots \ldots \ldots \ldots$ & 6,40 & $6,1 / 4$ & 7,35 & 7,61 & 7,62 & 7,64 \\
\hline Quantité de matière sc̀che consommée : & & & & & & \\
\hline par $100 \mathrm{~kg}$ de poids vif $\ldots \ldots \ldots \ldots$ & 1,97 & 1,87 & 1,78 & 1,76 & 2,01 & 1,96 \\
\hline par $\mathrm{kg}$ de gain $\ldots \ldots \ldots \ldots \ldots$ & 6,21 & 5,75 & 6,53 & 6,81 & 9,59 & $8,45^{*}$ \\
\hline Poids de carcasse chaude $(\mathrm{kg}) \ldots \ldots \ldots$ & 221 & 229 & 285 & 293 & 231 & 238 \\
\hline Poids de carcasse chaude $\times 100 \ldots$ & 67,5 & 67,9 & 70,1 & 69,2 & 67,2 & 67,1 \\
\hline $\begin{array}{l}\text { Poids vif vide } \\
\text { Poids du conteru digestif (p. } 100 \text { du poids } \\
\text { vif) } \ldots \ldots \ldots \ldots \ldots \ldots \ldots \ldots \ldots \ldots \ldots \ldots \ldots \ldots \ldots\end{array}$ & 12,8 & 11,7 & 12,5 & 12,8 & 14,6 & 14,7 \\
\hline $\begin{array}{l}\text { Dépôts adipeux périrénaux et précruraux } \\
\text { (p. } 100 \text { du poids de carcasse) } \ldots \ldots \ldots\end{array}$ & 2,08 & 2,06 & 2,19 & 1,71 & 3,98 & 3,78 \\
\hline $\begin{array}{l}\text { Dépôts adipeux dans la } 11^{\mathrm{e}} \text { côte désossée } \\
\quad(\text { p. } 100) \ldots \ldots \ldots \ldots \ldots \ldots \ldots \ldots \ldots \ldots \ldots \ldots \ldots\end{array}$ & 13,9 & 16,0 & 10,9 & 10,4 & & \\
\hline
\end{tabular}

* Différence significative $\mathrm{P}<0,05$.

Les bouvillons Frisons ont eu un gain de poids journalier assez faible, voisin de celui des animaux de $1^{\prime}$ expérience $n^{\circ} \mathrm{I}$, mais qui a été significativement accru, de I4 p. Ioo, par le traitement du maïs. L'efficacité alimentaire a été améliorée de I3 p. IOo. Ces différences traduisent une meilleure utilisation de l'énergie, d'autant plus importante que le croît de ces bouvillons devait être riche en lipides, comme le montre l'état d'engraissement des carcasses. En revanche, la cuisson du maïs n'a eu aucune influence sur les performances des taurillons. Ceux-ci ont eu un gain de poids journalier plus élevé que les bouvillons Frisons mais cependant relativement modeste; ils ont produit des carcasses plus maigres, surtout dans le cas des Charolais. 
La digestibilité des différents constituants de la ration a été la même pour les deux régimes : 66,8 et 66,6 pour la matière organique, 57,0 et 57,5 pour les matières cellulosiques, 60,3 et 60,7 pour les matière azotées, respectivement pour le régime témoin et pour le régime expérimental. La quantité d'azote retenue par jour a été légèrement plus élevée avec le maïs cuit (3I,2 au lieu de $28,4 \mathrm{~g}$ ) ainsi que la proportion d'acide propionique $d u$ jus de rumen prélevé par tubage I3 heures après le repas, mais la concentration en acides gras volatils du jus de rumen a été significativement plus faible $(59,8 \mathrm{mM} / 1$ au lieu de 74,8$)$.

Nous avons donc constaté une supériorité du maîs cuit avec les bouvillons Frisons mais aucune différence significative avec les taurillons Normands ou Charolais par rapport au maïs témoin. Nous avons pensé que l'effet de la cuisson ne pouvait pas apparaître complètement parce que les animaux avaient eu une ingestion de matière sèche faible (de 1,76 à $2,01 \mathrm{~kg}$ de matière sèche par Ioo $\mathrm{kg}$ de poids vif), probablement à cause de la qualité médiocre (faible teneur en azote) du foin.

TABLEAU 6

Expérience no 3: comparaison de la valeur nutritive $d u$ maïs grossièrement broyé et du maïs cuit

\begin{tabular}{|c|c|c|c|c|}
\hline & \multicolumn{2}{|c|}{ Maîs cru } & \multicolumn{2}{|c|}{ Mais cuit } \\
\hline & Période 1 & Période 2 & Période 1 & Période : \\
\hline Nombre d'animaux $\ldots \ldots \ldots \ldots \ldots \ldots$ & 9 & 9 & 9 & 9 \\
\hline Durée de la période expérimentale (j). & 49 & 71 & 49 & il \\
\hline Poids initial $(\mathrm{kg}) \ldots \ldots \ldots \ldots \ldots \ldots$ & 347 & 421 & 360 & 450 \\
\hline Poids final $(\mathrm{kg}) \ldots \ldots \ldots \ldots \ldots \ldots \ldots$ & 399 & 530 & 426 & 564 \\
\hline \multirow[t]{2}{*}{ Gain de poids journalier (g) $\ldots \ldots \ldots \ldots$} & 1 t 05 & 1535 & $1784 *$ & 1606 \\
\hline & \pm 212 & \pm 333 & \pm 234 & \pm 220 \\
\hline \multicolumn{5}{|l|}{ Quantité de matière sèche ingérée $(\mathrm{kg} / \mathrm{j})$ : } \\
\hline maïs $\ldots \ldots \ldots \ldots \ldots \ldots \ldots \ldots$ & $3, \geq 6$ & 4,51 & 3,62 & 4,61 \\
\hline tourteau d'arachide $\ldots \ldots \ldots \ldots$ & 0,32 & & 0,32 & \\
\hline Iuzerne $\ldots \ldots \ldots \ldots \ldots \ldots \ldots$ & 4,01 & 4,55 & 3,82 & 4,88 \\
\hline totale $\ldots \ldots \ldots \ldots \ldots \ldots \ldots \ldots$ & 7,59 & 9,02 & 7,76 & $9,4^{\prime} t$ \\
\hline \multicolumn{5}{|l|}{ Quantité de matière sèche consommée : } \\
\hline par $100 \mathrm{~kg}$ de poids vif $\ldots \ldots \ldots \ldots$ & 2,07 & 1,90 & 2,01 & 1,90 \\
\hline par $\mathrm{kg}$ de gain $\ldots \ldots \ldots \ldots \ldots$ & 5,87 & 6,10 & $4,43 *$ & 6,10 \\
\hline \multirow{3}{*}{$\begin{array}{l}\text { Poids de carcasse chaude }(\mathrm{kg}) \ldots \ldots \ldots \\
\frac{\text { Poids de carcasse chaude }}{\text { Poids vif vide }} \times 100 \ldots \ldots\end{array}$} & \multicolumn{2}{|c|}{$309 \pm 35$} & \multicolumn{2}{|c|}{$329 \pm 16$} \\
\hline & \multirow{2}{*}{\multicolumn{2}{|c|}{$69,9 \pm 0,8$}} & \multicolumn{2}{|c|}{$70,1+1,0$} \\
\hline & & & 70 & 1,0 \\
\hline $\begin{array}{l}\text { Dépôts adipeux périrénaux et précruraux } \\
\text { (p. } 100 \text { du poids de carcasse) } \ldots \ldots \ldots\end{array}$ & \multirow{2}{*}{\multicolumn{2}{|c|}{$2,6 \pm 0,7$}} & \multirow{2}{*}{\multicolumn{2}{|c|}{$2,6 \pm 0,4$}} \\
\hline Dépôts adipeux dans la $11^{\mathrm{e}}$ côte désossée & & & & \\
\hline (p. 100) $\ldots \ldots \ldots \ldots \ldots \ldots \ldots \ldots \ldots \ldots \ldots \ldots \ldots \ldots \ldots$ & \multicolumn{2}{|c|}{17} & \multicolumn{2}{|c|}{16} \\
\hline
\end{tabular}

* Différence significative $(P<0,01)$.

Période 1 : foin de luzerne sous forme normale.

Période 2 : farine de luzerne déshydratée. 


\section{Expérience no 3 (tabl. 6).}

Nous avons conservé le même protocole expérimental que dans l'expérience précédente mais en utilisant un foin de luzerne riche en azote $(I 7,4 \mathrm{p}$. roo de matières azotées totales) à la place du foin de prairie naturelle utilisé dans les essais précédents, et en modifiant le nombre et l'ordre des distributions (tab1. 2). Nous avons utilisé 24 taurillons Charolais ayant un potentiel de croissance élevé. Le lot témoin recevait du mais grossièrement broyé, le lot expérimental du maîs cuit pendant $45 \mathrm{mn}$. Au cours d'une première période de 7 semaines, le foin a été distribué sous forme normale, le tourteau étant mélangé au maìs. Au cours de la deuxième période (6 à $\mathrm{I} 2$ semaines selon les animaux), on a substitué au foin de luzerne, de la luzerne déshydratée, broyée et agglomérée ( 16,5 p. Ioo de matières azotées totales), et on a supprimé le tourteau par suite de l'augmentation des quantités de luzerne ingérées. La transition entre les deux périodes a duré deux semaines.

Pendant la première période, les animaux ont eu une vitesse de croissance très rapide, significativement $(\mathrm{P}<\mathrm{o}, \mathrm{or})$ plus élevée avec ceux qui recevaient le maïs cuit ( 784 au lieu de I $405 \mathrm{~g} / \mathrm{j}$ ). L'efficacité alimentaire a été excellente $(4,43 \mathrm{~kg}$ de matière sèche par $\mathrm{kg}$ de gain). Cependant, cette période n'a duré que 49 jours seulement et les animaux du lot expérimental ont consommé une proportion légèrement supérieure de maïs dans la ration (46,8 p. I00) que ceux du lot témoin (43, I p. IOO). Les différences se sont considérablement atténuées au cours de la deuxième période ( 606 contre I $535 \mathrm{~g} / \mathrm{j}$ ), en partie, parce que la croissance était déjà très élevée avec le maìs cru. Les animaux du lot expérimental, qui pesaient I3 $\mathrm{kg}$ de plus au départ ( ${ }^{1}$ ), ont fourni des carcasses plus lourdes (20 kg) que celles des animaux du lot témoin. On n'a pas noté de différence dans l'état d'engraissement.

Avec le mais cuit, la proportion d'acide acétique dans le jus de rumen a été plus importante $(\mathrm{P}<\mathrm{o}, \mathrm{OI})$ au détriment de l'acide butyrique et des acides gras supérieurs (tab1. 8). L'acidité totale du jus de rumen a été plus faible, seulement au cours de la première période. L'orientation des fermentations du rumen vers une production accrue d'acide propionique n'a eu lieu qu'au cours de la seconde période, lorsque les animaux ont reçu le foin condensé, qu'elle qu'ait été la nature du maïs.

\section{Influence du trempage suivi d'un séchage (expérience $\mathrm{n}^{\circ} 4$ )}

Les deux expériences précédentes ayant montré que la ctiisson pouvait, dans certains cas, améliorer légèrement la valeur nutritive du mais, nous avons essayé d'appliquer ces résultats en étudiant l'influence d'un traitement hydrothermique réalisé au stade industriel et pouvant être facilement caractérisé et reproduit (tab1. 7).

Deux lots de 12 taurillons (6 Charolais, 6 Salers $\times$ Charolais) ont reçu du maïs broyé et aggloméré. Dans le lot expérimental, 35 p. roo du maïs ont été traités industriellement par trempage du grain pendant 48 heures suivi d'un séchage sur rouleaux à $60-70^{\circ} \mathrm{C}$. Le reste de la ration était composé de foin de luzerne distribué sous forme normale. Le foin et le maîs ont été distribués chacun en deux repas.

Les animaux ont eu une croissance rapide et ont produit des carcasses lourdes en parfait état d'engraissement. Comme dans les essais précédents, les animaux

( ${ }^{1}$ Cette différence de poids initial provient de l'élimination de 2 animaux malades et d'un animal mort, ainsi que de leurs correspondants. 
recevant le maïs traité ont eu une croissance légèrement supérieure ( 1472 au lieu de I $4 \mathrm{I} 8 \mathrm{~g} / \mathrm{j})$ et une meilleure efficacité alimentaire $\left(6, \mathrm{I}_{3} \mathrm{~kg}\right.$ de matière sèche par $\mathrm{kg}$ de gain de poids au lieu de 6,52), mais les différences n'ont pas été significatives (tabl. 7). Les écarts auraient peut-être été plus importants si la proportion de maîs traité avait été plus élevée dans la ration expérimentale ; cette proportion a été limitée par le manque d'appétibilité du produit ainsi que par son coût. On n'a pas noté de différence dans l'état d'engraissement des carcasses.

TABLEAU 7

Expérience $n^{\circ} 4$ : comparaison de la valeur nutritive. du maïs grossièrement broyé et du mais ayant subi un traitement hydrothermique

\begin{tabular}{|c|c|c|}
\hline & Maïs témoin & Maïs traité \\
\hline Nombre d'animaux (1) & 11 & 11 \\
\hline Durée de l'expérience $(\mathrm{j}) \ldots \ldots \ldots \ldots$ & 91 & 91 \\
\hline Poids initial $(\mathrm{kg}) \ldots \ldots \ldots \ldots \ldots \ldots$ & 409 & 398 \\
\hline Poids final $(\mathrm{kg}) \ldots \ldots \ldots \ldots \ldots \ldots$ & 538 & 532 \\
\hline Gain de poids journalier (g) . . . . . . . & $1418 \pm 242$ & $1472 \pm 237$ \\
\hline 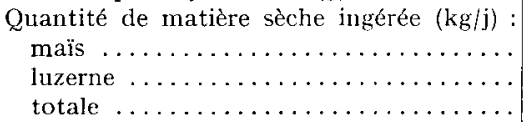 & $\begin{array}{l}4,59 \\
4,67 \\
9,25\end{array}$ & $\begin{array}{l}4,50 \\
4,52 \\
9,02\end{array}$ \\
\hline $\begin{array}{l}\text { Quantité de matière sèche consommée : } \\
\text { par } 100 \mathrm{~kg} \text { de poids vif } \ldots \ldots \ldots \ldots \ldots \\
\text { par } \mathrm{kg} \text { de gain } \ldots \ldots \ldots \ldots \ldots \ldots \ldots\end{array}$ & $\begin{array}{l}2,00 \\
6,52\end{array}$ & $\begin{array}{l}1,99 \\
6,13\end{array}$ \\
\hline Poids de carcasse chaude (kg) . . . . . . & $318 \pm 31$ & $311 \pm 33$ \\
\hline$\frac{\text { Poids de carcasse chaude }}{\text { Poids vif vide }} \times 100, \ldots \ldots$ & $69,2 \pm 1,0$ & $69,0 \pm 1,5$ \\
\hline $\begin{array}{c}\text { Dépòts adipeux périrénaux (p. } 100 \text { du } \\
\text { poids de carcasse) } \ldots \ldots \ldots \ldots \ldots \ldots \ldots \ldots\end{array}$ & $2,1 \pm 0,5$ & $2,1 \pm 0, \mathbf{f}$ \\
\hline
\end{tabular}

(1) Un couple d'animaux a dû être éliminé, l'un d'entre eux étant malade.

\section{DISCUSSION}

Les traitements que nous avons utilisés ont modifié notablement la structure de l'amidon, surtout dans le cas du mais traité de l'expérience $\mathrm{n}^{\circ} 3$, comme l'indiquent les différentes courbes d'amylolyse in vitro que nous avons effectuées (fig. I). Cependant, ces modifications n'ont pas orienté les fermentations du rumen dans un sens favorable à une meilleure utilisation de l'énergie (augmentation de la production d'acide propionique et butyrique, diminution de la production d'acide acétique). En effet, la composition du mélange d'acides gras volatils du jus de rumen n'a jamais été modifiée de façon significative par le traitement du maïs, sauf dans le cas de l'expérience $n^{\circ} 3$ au cours de laquelle la proportion d'acide acétique a augmenté tandis que diminuait la proportion d'acide butyrique. Ces résultats ne correspondent pas à 1'hypothèse initiale, déduite des travaux de SHAw et al. (I960). Toutefois, si dans 
l'expérience $n^{\circ} 3$, nous comparons (tabl. 8) la teneur et la composition en acides gras volatils du jus de rumen des animaux recevant le mais cru et le foin normal avec celles des animaux recevant le mais cuit et le foin condensé, nous observons une augmentation de la proportion et de la production d'acide propionique avec des régimes analogues à ceux utilisés par SHAw et al. (I960) (tabl. I). Mais cette différence doit provenir surtout de l'action du broyage et du pressage du foin (BÉRANGER et JARRIGE, I962) et non de l'action du traitement hydrothermique du maïs. Nos résultats sont généralement en accord avec ceux de nombreux auteurs (Thompson, BRAdLEY et LITTLE, I965 ; DE BIE et WoODS, I964) qui, par ailleurs, n'ont observé qu'une légère amélioration de la valeur nutritive du mais traité.

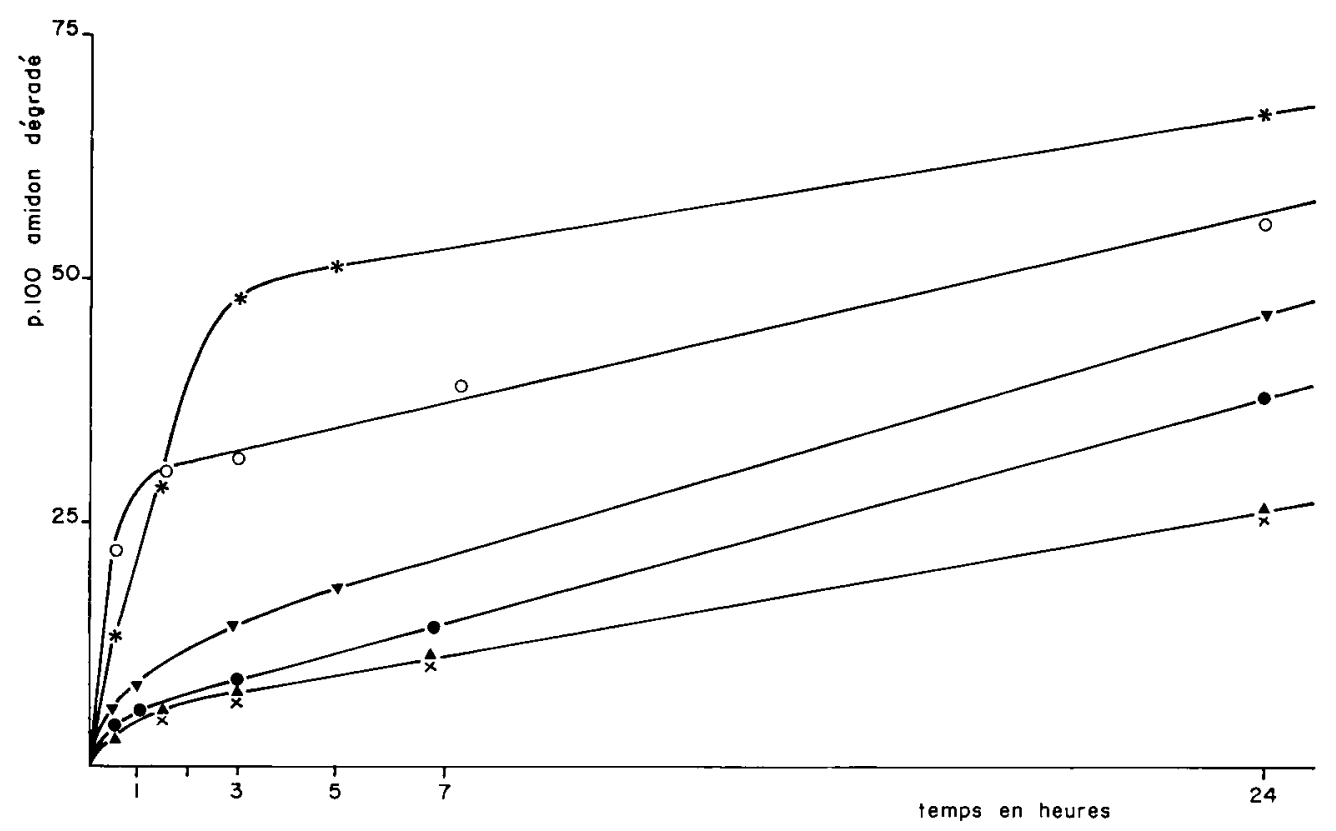

FIG. 1. - Cinétique de la dégradation enzymatique du mä̈s en fonction du traitement technologique étudié

(L'amylolyse a été effectuée à $37^{\circ} \mathrm{C}$ en présence de $3 \mathrm{p}$. Ioo d'amylase bactérienne par rapport au substrat ; les produits d'hydrolyse (glucides alcoolosolubles dans l'alcool à 80 p. 1oo) ont été dosés par la méthode à l'anthrone).

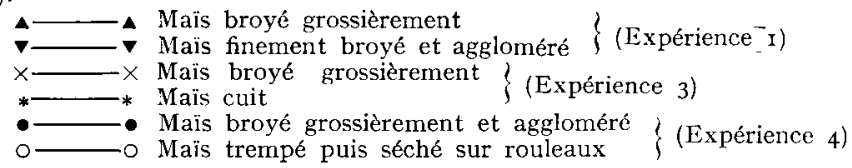

En revanche, le traitement technologique du mais peut modifier les quantités d'amidon digérées respectivement dans le rumen et dans le reste du tube digestif (ØRSKov, FraSER et KAY, I969). Au moment de l'abattage, nous avons observé chez les animaux recevant du maïs cru, qu'une quantité importante d'amidon échappait aux fermentations du rumen pour être digérée sans doute dans l'intestin grêle, 


\begin{tabular}{|c|c|c|c|c|c|c|}
\hline \multirow{2}{*}{$\begin{array}{c}3 \\
30 \\
5 \\
3 \\
5 \\
3 \\
0 \\
0\end{array}$} & \multirow{5}{*}{ 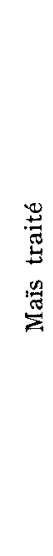 } & 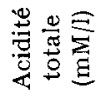 & $\bar{s}$ & 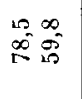 & 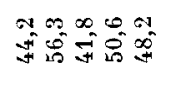 & 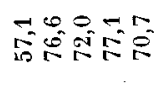 \\
\hline & & $\begin{array}{l}* \\
\wedge\end{array}$ & $\stackrel{\infty}{\infty}$ & 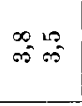 & $\begin{array}{lll}n & \infty \\
\text { oi } & \infty\end{array}$ & 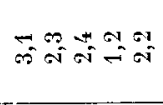 \\
\hline $\begin{array}{l}\text { है } \\
\text { है } \\
\text { "ूँ }\end{array}$ & & $u^{\prime \prime}$ & $\stackrel{+}{\infty}$ & $\begin{array}{l}\stackrel{0}{=} \\
\therefore \stackrel{\infty}{\oplus}\end{array}$ & 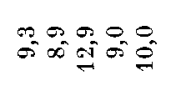 & 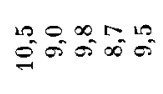 \\
\hline है & & i & $\stackrel{\infty}{\stackrel{\infty}{*}}$ & $\begin{array}{l}+4 \\
g^{2}\end{array}$ & 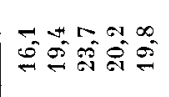 & 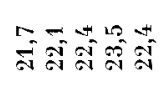 \\
\hline 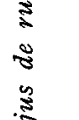 & & $e^{20}$ & 8 & $\frac{1}{80}$ & 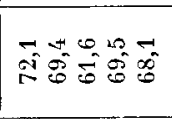 & 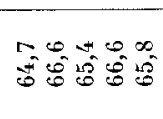 \\
\hline 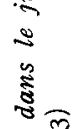 & & 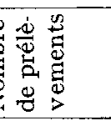 & 0 & 106 & 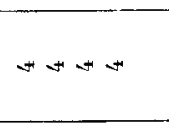 & $-\mu-$ \\
\hline 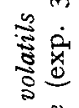 & & 莺。若 & $\underset{\infty}{\stackrel{5}{\infty}}$ & 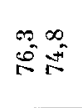 & $\begin{array}{lll}0 & = \\
8 \\
8\end{array}$ & 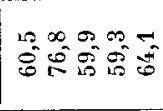 \\
\hline 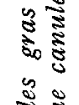 & .5 & $\begin{array}{l}U^{*} \\
\wedge\end{array}$ & 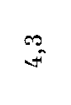 & 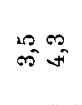 & 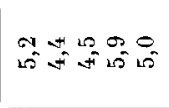 & 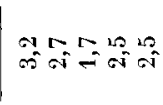 \\
\hline 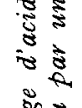 & 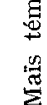 & $\Xi^{*}$ & : & $\begin{array}{l}\infty \\
\infty \\
= \\
=\end{array}$ & 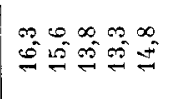 & 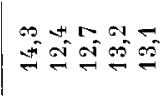 \\
\hline 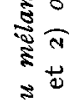 & & $\omega^{\infty}$ & $\stackrel{\theta}{0}$ & $\Rightarrow$ & 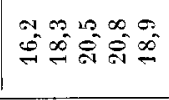 & 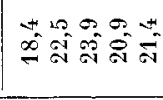 \\
\hline$\underset{\bar{g}}{\dot{\vec{x}}}$ & & $\omega^{N}$ & 8 & $\begin{array}{ll}0 & 0 \\
0 & 0 \\
0 & 0 \\
0 & 0\end{array}$ & 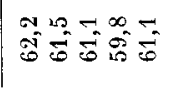 & 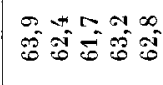 \\
\hline 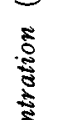 & & 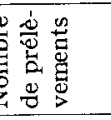 & $\infty$ & 00 & Hot & Th स स स \\
\hline 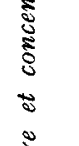 & & 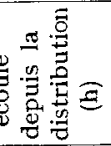 & & $\infty=$ & $\tan 20$ & $-\infty \cos$ \\
\hline 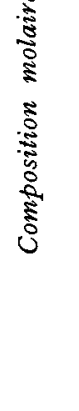 & & & 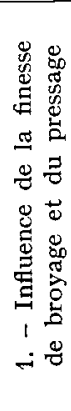 & 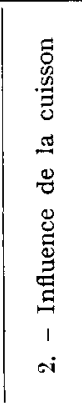 & 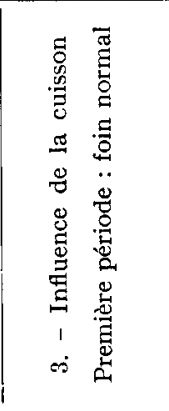 & 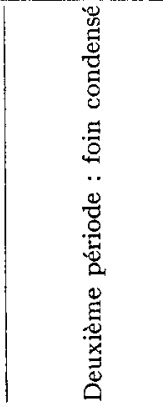 \\
\hline
\end{tabular}


sous forme de glucose ; chez les animaux recevant le mais traité, 1'amidon était digéré en quasi-totalité dans le rumen sous forme d'acides gras volatils (THIVEND et BÉRANGER). Or, on sait que le glucose est mieux utilisé pour l'engraissement des animaux que les acides gras volatils (ARMSTrong et BLAXTER, I96I). Par conséquent, l'effet bénéfique éventuel du traitement du maïs qui pourrait résulter d'une production légèrement supérieure d'acide propionique, a pu être contrebalancé par une diminution de la production de glucose, ce qui expliquerait au moins en partie, la faible augmentation des performances des animaux recevant le maïs traité au cours de nos essais.

Nous avons réduit en outre les possibilités d'extérioriser l'influence des traitements étudiés en limitant à $50 \mathrm{p}$. Ioo la proportion de céréales dans la ration et en utilisant, au cours des deux premières expériences, un foin de prairie naturelle de qualité médiocre, distribué seul, à plusieurs heures d'intervalle du repas de maīs. L'appétit des animaux a donc été limité par la faible vitesse de digestion des aliments qui aurait été sans doute plus importante si 1'azote et les glucides avaient été apportés simultanément. En utilisant un foin de luzerne de bonne qualité, distribué avec le mais en deux repas par jour, nous avons observé (expérience $n^{\circ} 3$ ) des différences importantes dans la vitesse de croissance et l'efficacité alimentaire en faveur des animaux qui recevaient le maïs traité mais seulement pendant la première partie de l'essai, qui a d'ailleurs eu une durée relativement courte. Au cours de l'expérience no 4 , le faible pourcentage de maîs traité a sans doute empêché d'obtenir, dans les mêmes conditions, des résultats aussi nets.

Si nos résultats ne permettent pas de mettre clairement en évidence l'influence des traitements du mais, ils montrent cependant dans chaque expérience, une tendance positive en faveur du lot recevant le maïs traité. Cette légère amélioration pourrait être due vraisemblablement à une meilleure utilisation de l'azote du régime. COLENBRANDER et al. (I967, I968) ont en effet montré que le traitement technologique de la céréale réduit l'ammoniogenèse dans le rumen; cette amélioration de l'utilisation de l'ammoniaque est sans doute d'autant plus importante que l'azote et les glucides facilement fermentescibles provenant de l'amidon traité sont apportés simultanément (expérience $n^{\circ} 3$ ). Cependant, l'influence du traitement technologique sur l'utilisation des protéines de la ration est fonction de l'état de dégradation de l'amidon et probablement aussi de la nature de l'azote. D'après ZELTER et CHARLETLERY (I969), l'orge déshydratée entraîne chez les bovins, une meilleure rétention azotée que l'orge ensilée lorsque la ration est supplémentée en urée. En revanche, $\mathrm{KAY}$ et al. (I966) n'observent aucune différence dans l'utilisation de l'azote d'un régime à base d'orge broyée, cuite à la vapeur, ou conservée humide. WARD et MORRILI (I966) utilisant un traitement hydrothermique sévère provoquant la gélatinisation complète de l'amidon du sorgho ne constatent pas d'amélioration de la rétention azotée; en revanche, ils observent une diminution du CUD des matières azotées, probablement par suite d'une insolubilisation partielle des protéines de la céréale au cours du traitement. MUDD et PERRY (I969) aboutissent aux mêmes conclusions en étudiant l'influence de la gélatinisation de l'amidon du mais, sur les variations de la digestibilité de la ration.

En conclusion, nous avons presque toujours constaté une tendance à l'amélioration du gain de poids vif des animaux et de l'efficacité alimentaire de la ration en faveur du maîs traité mais elle a été rarement significative. La légère augmentation de l'efficacité alimentaire mesurée pour l'ensemble de la ration devient cependant 
plus importante si on admet qu'elle ne provient que de l'augmentation de la valeur nutritive de la céréale. Avec les rations ne comportant pas plus de $50 \mathrm{p}$. roo de maîs, il ne semble pas que l'amélioration de l'efficacité alimentaire puisse être supérieure à Io p. IOo. Dans ces conditions, seul le coût du traitement peut décider de l'opportunité d'utiliser cette céréale traitée dans l'alimentation du ruminant.

Reçu pour publication en décembre 1971.

\section{SUMMARY}

\section{EFFECT OF MECHANICAL AND HEAT-MOISTURE, TREATMENTS}

\section{ON THE NUTRITIVE VALUE OF MAIZE FOR YOUNG FATTENING BEEF CATTLE}

I. Four fattening trials with young beef cattle were carried out to study the effect of three simple treatments on the nutritive value of maize. The processing methods used were the following : fine grinding (size $3 \mathrm{~mm}$ ) followed by pelleting (trial $\mathrm{I}$ ), cooking for $30-45$ minutes (trials 2 and 3 ) and soaking for $48 \mathrm{hrs}$ followed by roller drying at $70^{\circ} \mathrm{C}$ (trial 4 ). In each case, the experimental group receiving treated maize was compared with a control group fed coarsely ground maize. The diets were composed of $50 \mathrm{p}$. Ioo maize, hay and some oil cake. The nature and amount of hay as well as the order of distribution of the different feeds varied according to trials (table. 2).

2. 126 animals of the Frisian, Norman, Charolais or Charolais $\times$ Salers breeds (table. 2) were fattened for periods of roo to 150 days. In all trials, i 2 animals of each group were fed individually and the others in groups of 5 or 6 animals. The amount of maize offered to all animals was I-I.5 kg dry matter per I oo $\mathrm{kg}$ live weight. The quantity of hay given almost reached the maximum intake level, but did not exceed $50 \mathrm{p}$. Ioo of the dry matter of the diet. For each trial, we measured daily feed intake, growth rate of the animals, weight and fatness of the carcasses, and in some cases, digestibility of the diet and composition of the volatile fatty acid mixture of the rumen fluid.

3. During trial number $\mathbf{r}$, no significant difference was noticed between the animals receiving coarsely ground maize (controls) and those fed finely ground and pelleted maize as regards live weight carcass gain, fattening state, feed efficiency (table 4) and proportion of different acids in the volatile fatty acid mixture of the rumen fluid (table 8).

4. The cooked maize (trial 2, table 5) was significantly better than the control maize when fed on young Frisian steers, but not with young Norman and Charolais bulls. The effect of cooking could perhaps not come out clearly as the appetite of the animals was small, probably because of the poor quality of the hay and the order of distribution of the meals (tables 2 and 3 ). During trial 3 (table 6 ), the animals received protein rich lucerne hay offered at the same time as the maize. In the first seven weeks of experiment, the growth rate and feed efficiency of the animals receiving treated maize were higher $(\mathrm{P}<\mathrm{o.oI})$ than those of the control group. The differences became smaller during the second period when lucerne hay was replaced by dehydrated, ground and pelletted lucerne.

5. When one third of the maize ration was made of soaked, then roller dried maize (trial 5 , table 7), no effect of the treatment could be noticed.

6. The small influence of the three treatments on the nutritive value of maize in young beef cattle may be explained as follows :

- the treatments applied did not affect the rumen fermentations favourably towards a better energy utilization, since the composition of the volatile fatty acid mixture of the rumen fluid was never significantly modified (no increase of the propionic acid production). However, these treatments greatly altered the structure of starch (fig. I). On the other hand, the amount of starch broken down in the small intestine was lower with treated maize than with coarsely ground maize.

- The possibilities of demonstrating the effect of the treatments studied was reduced by limiting the proportion of cereals in the diet to $50 \mathrm{p}$. Ioo, and by using, during the first two trials, poor quality grass hay, offered several hours after the maize meal.

iiven though the advantage of treating cereals is not obvious from our results, a positive trend in favour of the group receiving treated maize can be noticed in each trial, probably due to a better use of the diet proteins. 


\section{RÉFÉRENCES BIBL,IOGRAPHIQUES}

Armstrong D. G., Blaxter K. L., I96I. The utilization of the energy of carbohydrates by ruminant (in 2 nd Symposium on energy metabolism Wageningen p. 178).

Arnett D. W., Bradley N. W., r961. Effects of pelleted, flaked, and ground corn and pelleted distillers dried grains with solubles on digestibility of fattening rations and feed lot performance of beef steers. J. Anim. Sci., 20, 396 (Abstr.).

BERANGer C., JARRIGE R., I962. Utilisation des aliments broyés et agglomérés par les bovins. I. Comparaison du foin de luzerne normal et du foin de luzerne broyé dans l'alimentation du bœuf à l'engrais. Ann. Zootech., 11, 273-294.

Christian K. R., Coup M. R., I954. Measurement of feed intake by grazing cattle and sheep. VI. Determination of chromic oxide in faeces. New. Z. J. Sci. Tech., 36, 328-330.

Colenbrander V. F., Bartley E. E., Morrill J. L., Deyoe C. W., Pfost H. B., ig67. Feed processing. II. Effect of feeding expanded grain and finely ground hay on milk composition, yield, and rumen metabolism. J. Dairy. Sci., 50, 1966-1972.

Colenbrander V. F., Bartley E. E., Morrill J. L., Deyoe C. W., Meyer R. M., Pfost H. B., 1968. Feed processing. IV. Effect of feeding expanded grain and ground hay on concentrations of rumen ammonia. J. Dairy. Sci., 51, 1974-1977.

Davis R. E., Oltjen R. R., Bond J., I963. High concentrate studies with beef cattle. J. Anim. Sci., 22, 640-643.

De Bie W. H., Woods W., ig64. Rumen fermentation and animal performance as influenced by gelatinized corn and enzyme supplementation. J. Anim. Sci, 23, 872. (Abstr.).

Ensor W. L., Shaw J. C., Tellechea H. F., 1959. Special diets for production of low fat milk and more efficient gains in body weight. J. Dairy Sci., 42, I89-19I.

Geay Y., Beranger C., I969. Estimation de la composition de la carcasse de jeunes bovins à partir de la composition d'un morceau monocostal au niveau de la II eôte. Ann. Zootech. , 18, 65-77.

James A. T., Martin J. P., r95I. Gas liquid partition chromatography : microestimation of volatile fatty acids from formic acid to dodecanoic acid. Biochem. $J ., 50,679-690$.

Johnson D. E., Matsushima J. K., KNox K. L., I968. Utilization of flaked VS cracked corn by steers with observations on starch modification. J. Anim. Sci., 27, I43I-I 437.

Kay M., Preston T. R., Macleod N. A., Philip E. B., Ig66. Intensive beef production. VII. The effect of nitrogen retention of diets containing barley processed by four different methods. Anim. Prod., 8, 39-42.

Mudd C. A., Perry T. W., I969. Raw cracked VS expanded gelatinized corn for beef cattle. J. Anim. Sci., 28, 822-826.

Newland H. W., Magee, W. T., Branaman G. A., Blakeslee L. H., ig62. Effect of heat processing and pelleting corn for steers and lambs. J. Anim. Sci, 21, 7II-7I5.

Ørskov E. R., Fraser C., KAY R. N. B., I969. Dietary factors influencing the digestion of starch in the rumen and small and large intestine of early weaned lambs. Br. J. Nutr., 23, 217-226.

Phillipson A. T., I952. The fatty acids present in the rumen of lambs fed on a flaked maize ration. Br. J. Nutr., 6, 190-198.

Shaw J. C., Ensor W. L., Tellechea H. F., Lee S. D., 1960. Relation of diet to rumen volatile fatty acids, digestibility, efficiency of gain and degree of insaturation of body fat in steers. J.Nutr., 71, 203-208.

Thivend P., Béranger C., Résultats non publiés.

Thompson J. T., Bradley N. W., Little C. O., I965. Ruminal volatile fatty acid concentration and performance of steers fed different levels and forms of hay and grain. J.Anim. Sci., 24, I I 79-I I83.

WARD G. M., Morrill J. L., 1966. Digestibility and nitrogen utilization of rolled and moist-heat-treated sorghum grain. J. Dairy Sci., 49, 392-394.

Woods W., Luther R., I962. Further observations on the effect of physical preparation of the ration on volatile fatty acids production. J, Anim. Sci., 21, 809-814.

Zelter S. Z., Charlet-Lery G., I969. Forme de présentation de l'aliment énergétique (orge immature) et taux de rétention de $\mathrm{N}$-uréique chez le taurillon en croissance. C. R. Acad. Sci., 269 D., roo7-1oro. 